\title{
Multiple periodic orbits of high-dimensional differential delay systems
}

Zhongmin Sun ${ }^{1}$, Weigao Ge $\mathrm{G}^{2}$ and Lin $\mathrm{Li}^{2^{*}}$

\section{"Correspondence:}

545713048@qq.com

${ }^{2}$ School of Mathematics and

Statistics, Beijing Institute of

Technology, Beijing, P.R. China

Full list of author information is

available at the end of the article

\section{Abstract}

In this paper, we consider differential delay systems of the form

$$
x^{\prime}(t)=-\sum_{s=1}^{2 k-1}(-1)^{s+1} \nabla F(x(t-s))
$$

in which the coefficients of the nonlinear terms with different hysteresis have different signs. Such systems have not been studied before. The multiplicity of the periodic orbits is related to the eigenvalues of the limit matrix. The results provide a theoretical basis for the study of differential delay systems.

MSC: 34K13; 58E50

Keywords: Differential delay system; Periodic orbits; Critical point theory; Variational method

\section{Introduction}

We consider the asymptotically linear differential delay system

$$
x^{\prime}(t)=-\sum_{s=1}^{2 k-1}(-1)^{s+1} \nabla F(x(t-s)),
$$

where

$$
x \in R^{N}, \quad F \in C^{1}\left(R^{N}, R\right), \quad \nabla F(-x)=-\nabla F(x),
$$

and there are real symmetric matrices $A_{0}, A_{\infty} \in R^{N \times N}$ such that

$$
\nabla F(x)=A_{0} x+\circ(|x|), \quad|x| \rightarrow 0, \quad \nabla F(x)=A_{\infty} x+\circ(|x|), \quad|x| \rightarrow \infty .
$$

In the past several decades, many papers [1-16] have studied the existence of periodic solutions of delay differential equations. In 1974, Kaplan and Yorke [15] studied the multiple periodic solutions of the equations

$$
x^{\prime}(t)=-f(x(t-1))
$$

(c) The Author(s) 2019. This article is distributed under the terms of the Creative Commons Attribution 4.0 International License (http://creativecommons.org/licenses/by/4.0/), which permits unrestricted use, distribution, and reproduction in any medium, provided you give appropriate credit to the original author(s) and the source, provide a link to the Creative Commons license, and indicate if changes were made. 
and

$$
x^{\prime}(t)=-f(x(t-1))-f(x(t-2))
$$

by transforming them respectively into associated systems of ordinary differential equations and then making analysis by qualitative approaches. Meanwhile, they guessed that there should exist $2(n+1)$-periodic solutions to the equation

$$
x^{\prime}(t)=-\sum_{i=1}^{n} f(x(t-i)),
$$

where $f \in C^{0}(R, R)$ with $f(-x)=-f(x), x f(x)>0, x \neq 0$. This was proved in [17]. On the basis of this work, Fei $[3,4]$ studied the multiple periodic solutions of differential delay equations via Hamiltonian systems. Li and He [10-12] studied the multiple solutions by an asymptotically linear Hamiltonian system. Guo and $\mathrm{Yu}[13,14]$ gave some multiple results for periodic solutions via critical point theory.

In this paper, our main purpose is to study system (1.1), in which the coefficients of nonlinear terms corresponding to different hysteresis have different signs, which is an extension of [3]. To construct the even functional, the variation structure here is much simpler since we do not transform system (1.1) into a $2 k N$-dimensional system. At the same time, according to the variational method and the method of Kaplan-Yorke coupling system, we get an exact counting method of the number of $4 k$-periodic orbits. Moreover, our results are easier to examine by introducing the eigenvalues and eigenvectors of the matrices $A_{\infty}$ and $A_{0}$.

Let

$$
\alpha_{1} \leq \alpha_{2} \leq \cdots \leq \alpha_{N} \quad \text { and } \quad \beta_{1} \leq \beta_{2} \leq \cdots \leq \beta_{N}
$$

be the eigenvalues of $A_{0}$ and $A_{\infty}$, respectively, and let $u_{1}, u_{2}, \ldots, u_{N}$ and $v_{1}, v_{2}, \ldots, v_{N}$ be the corresponding unit eigenvectors in space. For convenience, we make the following assumptions:

$\left(f_{1}\right) F$ satisfies (1.2) and (1.3),

$\left(f_{2}\right)$ there are $M>0$ and a function $r \in C^{0}\left(R^{+}, R^{+}\right)$satisfying $r(s) \rightarrow \infty$ and $r(s) / s \rightarrow 0$ as $s \rightarrow \infty$ such that

$$
\left|F(x)-\frac{1}{2}\left(A_{\infty} x, x\right)\right|>r(|x|)-M
$$

$\left(f_{3}^{ \pm}\right) \pm\left[F(x)-\frac{1}{2}\left(A_{\infty} x, x\right)\right]>0,|x| \rightarrow \infty$,

$\left(f_{4}^{ \pm}\right) \pm\left[F(x)-\frac{1}{2}\left(A_{0} x, x\right)\right]>0,0<|x| \ll 1$.

\section{Variational structure}

Let

$$
\begin{aligned}
\widehat{X} & =\left\{x \in L^{2}: x(t-2 k)=-x(t)\right\} \\
& =\left\{\sum_{i=0}^{\infty}\left(a_{i} \cos \frac{(2 i+1) \pi t}{2 k}+b_{i} \sin \frac{(2 i+1) \pi t}{2 k}\right): a_{i}, b_{i} \in R^{N}\right\},
\end{aligned}
$$




$$
\begin{gathered}
X=\mathrm{cl}\left\{\sum_{i=0}^{\infty}\left(a_{i} \cos \frac{(2 i+1) \pi t}{2 k}+b_{i} \sin \frac{(2 i+1) \pi t}{2 k}\right):\right. \\
\left.a_{i}, b_{i} \in R^{N}, \sum_{i=0}^{\infty}(2 i+1)\left(a_{i}^{2}+b_{i}^{2}\right)<\infty\right\} \subset \widehat{X},
\end{gathered}
$$

and define $P: X \rightarrow L^{2}$ by

$$
\begin{aligned}
\operatorname{Px}(t) & =P\left(\sum_{i=0}^{\infty}\left(a_{i} \cos \frac{(2 i+1) \pi t}{2 k}+b_{i} \sin \frac{(2 i+1) \pi t}{2 k}\right)\right) \\
& =\sum_{i=0}^{\infty}(2 i+1)\left(a_{i} \cos \frac{(2 i+1) \pi t}{2 k}+b_{i} \sin \frac{(2 i+1) \pi t}{2 k}\right)
\end{aligned}
$$

and the inverse of $P$ as

$$
P^{-1} x(t)=\sum_{i=0}^{\infty} \frac{1}{2 i+1}\left(a_{i} \cos \frac{(2 i+1) \pi t}{2 k}+b_{i} \sin \frac{(2 i+1) \pi t}{2 k}\right) .
$$

Define

$$
\begin{aligned}
& \langle x, y\rangle=\int_{0}^{4 k}(P x(t), y(t)) d t, \quad\|x\|=\sqrt{\langle x, x\rangle} \\
& \langle x, y\rangle_{2}=\int_{0}^{4 k}(x(t), y(t)) d t, \quad\|x\|_{2}=\sqrt{\langle x, x\rangle_{2}} .
\end{aligned}
$$

Then $(X,\|\cdot\|)$ is an $H_{4 k}^{\frac{1}{2}}\left([0,4 k], R^{N}\right)$ space.

For system (1.1), define the following functional $\Phi: X \rightarrow R$ :

$$
\Phi(x)=\frac{1}{2}\langle L x, x\rangle+\int_{0}^{4 k} F(x(t)) d t
$$

where

$$
L x=-P^{-1} \sum_{s=1}^{2 k-1} x^{\prime}(t-s)
$$

Let

$$
X(i)=\left\{x(t)=a_{i} \cos \frac{(2 i+1) \pi t}{2 k}+b_{i} \sin \frac{(2 i+1) \pi t}{2 k}: a_{i}, b_{i} \in R^{N}\right\} .
$$

Then we have

$$
X=\sum_{h=0}^{\infty}\left[\sum_{i=0}^{k-1}(X(2 h k+i)+X(2 h k+2 k-i-1))\right] .
$$

On the basis of Theorem 1.4 in [18], we can get that the differential of $\Phi$ satisfies

$$
\Phi^{\prime}(x)=L x+K(x)
$$

where $K(x)=P^{-1} \nabla F(x)$. 
For convenience of further calculations, we can also make a more detailed division of space $X$ by introducing the eigenvalues and eigenvectors mentioned before.

Suppose that

$$
\begin{aligned}
& X_{0 j}(i)=\left\{x(t)=\left(a_{j} \cos \frac{(2 i+1) \pi t}{2 k}+b_{j} \sin \frac{(2 i+1) \pi t}{2 k}\right) u_{j}: a_{j}, b_{j} \in R, j=1,2, \ldots, N\right\}, \\
& X_{\infty j}(i)=\left\{x(t)=\left(a_{j} \cos \frac{(2 i+1) \pi t}{2 k}+b_{j} \sin \frac{(2 i+1) \pi t}{2 k}\right) v_{j}: a_{j}, b_{j} \in R, j=1,2, \ldots, N\right\} .
\end{aligned}
$$

Then we have

$$
X(i)=\sum_{j=1}^{N} X_{\infty j}(i)=\sum_{j=1}^{N} X_{0 j}(i)
$$

and

$$
X=\sum_{i=0}^{\infty} X(i)=\sum_{i=0}^{\infty} \sum_{j=1}^{N} X_{\infty j}(i)=\sum_{i=0}^{\infty} \sum_{j=1}^{N} X_{0 j}(i)
$$

Therefore from (2.2) we find that if

$$
x(t)=\sum_{i=0}^{\infty}\left(a_{i} \cos \frac{(2 i+1) \pi t}{2 k}+b_{i} \sin \frac{(2 i+1) \pi t}{2 k}\right),
$$

then

$$
\begin{aligned}
\langle L x, x\rangle= & -\sum_{i=0}^{\infty}(2 i+1) \pi\left(a_{i}^{2}+b_{i}^{2}\right) \cot \frac{(2 i+1) \pi}{4 k} \\
= & \sum_{h=0}^{\infty}\left[-\sum_{i=0}^{k-1}(4 h k+2 i+1) \pi\left(a_{2 h k+i}^{2}+b_{2 h k+i}^{2}\right) \cot \frac{(2 i+1) \pi}{4 k}\right. \\
& \left.+\sum_{i=0}^{k-1}(4 h k+4 k-2 i-1) \pi\left(a_{2 h k+2 k-i-1}^{2}+b_{2 h k+2 k-i-1}^{2}\right) \cot \frac{(2 i+1) \pi}{4 k}\right] .
\end{aligned}
$$

On the other hand, when

$$
\begin{aligned}
x & \in X_{\infty j}=\sum_{i=0}^{\infty} X_{\infty j}(i) \\
& =\sum_{i=0}^{\infty}\left\{x(t)=\left(a_{j} \cos \frac{(2 i+1) \pi t}{2 k}+b_{j} \sin \frac{(2 i+1) \pi t}{2 k}\right) v_{j}: a_{j}, b_{j} \in R, j=1,2, \ldots, N\right\},
\end{aligned}
$$

we have

$$
\begin{aligned}
\left\langle P^{-1} A_{\infty} x, x\right\rangle & =\sum_{i=0}^{\infty} 2 k \beta_{j}\left(a_{i}^{2}+b_{i}^{2}\right) \\
& =\sum_{h=0}^{\infty}\left[\sum_{i=0}^{k-1} 2 k \beta_{j}\left(a_{2 h k+i}^{2}+b_{2 h k+i}^{2}\right)+\sum_{i=0}^{k-1} 2 k \beta_{j}\left(a_{2 h k+2 k-i-1}^{2}+b_{2 h k+2 k-i-1}^{2}\right)\right] .
\end{aligned}
$$


Then we can get

$$
\begin{aligned}
\langle(L+ & \left.\left.P^{-1} A_{\infty}\right) x, x\right\rangle \\
= & 2 k \sum_{j=1}^{N} \sum_{h=0}^{\infty}\left[\sum_{i=0}^{k-1}\left(-\frac{(4 h k+2 i+1) \pi}{2 k} \cot \frac{(2 i+1) \pi}{4 k}+\beta_{j}\right)\left(a_{2 l k+i}^{2}+b_{2 h k+i}^{2}\right)\right. \\
& \left.+\sum_{i=0}^{k-1}\left(\frac{(4 h k+4 k-2 i-1) \pi}{2 k} \cot \frac{(2 i+1) \pi}{4 k}+\beta_{j}\right)\left(a_{2 h k+2 k-i-1}^{2}+b_{2 h k+2 k-i-1}^{2}\right)\right] .
\end{aligned}
$$

Similarly,

$$
\begin{aligned}
\langle(L+ & \left.\left.P^{-1} A_{0}\right) x, x\right\rangle \\
= & 2 k \sum_{j=1}^{N} \sum_{h=0}^{\infty}\left[\sum_{i=0}^{k-1}\left(-\frac{(4 h k+2 i+1) \pi}{2 k} \cot \frac{(2 i+1) \pi}{4 k}+\alpha_{j}\right)\left(a_{2 h k+i}^{2}+b_{2 h k+i}^{2}\right)\right. \\
& \left.+\sum_{i=0}^{k-1}\left(\frac{(4 h k+4 k-2 i-1) \pi}{2 k} \cot \frac{(2 i+1) \pi}{4 k}+\alpha_{j}\right)\left(a_{2 h k+2 k-i-1}^{2}+b_{2 h k+2 k-i-1}^{2}\right)\right] .
\end{aligned}
$$

\section{Division of space $X$ and lemmas}

Let

$$
\begin{aligned}
X_{\infty}^{+}= & \sum_{j=1}^{N}\left\{X_{\infty j}(2 h k+i): h \geq 0,0 \leq i \leq k-1,-\frac{(4 h k+2 i+1) \pi}{2 k} \cot \frac{(2 i+1) \pi}{4 k}+\beta_{j}>0\right\} \\
& \cup \sum_{j=1}^{N}\left\{X_{\infty j}(2 h k+2 k-i-1):\right. \\
& \left.h \geq 0,0 \leq i \leq k-1, \frac{(4 h k+4 k-2 i-1) \pi}{2 k} \cot \frac{(2 i+1) \pi}{4 k}+\beta_{j}>0\right\}, \\
X_{\infty}^{-}= & \sum_{j=1}^{N}\left\{X_{\infty j}(2 h k+i): h \geq 0,0 \leq i \leq k-1,-\frac{(4 h k+2 i+1) \pi}{2 k} \cot \frac{(2 i+1) \pi}{4 k}+\beta_{j}<0\right\} \\
& \cup \sum_{j=1}^{N}\left\{X_{\infty j}(2 h k+2 k-i-1):\right. \\
& \left.h \geq 0,0 \leq i \leq k-1, \frac{(4 h k+4 k-2 i-1) \pi}{2 k} \cot \frac{(2 i+1) \pi}{4 k}+\beta_{j}<0\right\}, \\
X_{0}^{+}= & \sum_{j=1}^{N}\left\{X_{0 j}(2 h k+i): h \geq 0,0 \leq i \leq k-1,-\frac{(4 h k+2 i+1) \pi}{2 k} \cot \frac{(2 i+1) \pi}{4 k}+\alpha_{j}>0\right\} \\
& \cup \sum_{j=1}^{N}\left\{X_{0 j}(2 h k+2 k-i-1):\right. \\
& \left.h \geq 0,0 \leq i \leq k-1, \frac{(4 h k+4 k-2 i-1) \pi}{2 k} \cot \frac{(2 i+1) \pi}{4 k}+\alpha_{j}>0\right\},
\end{aligned}
$$




$$
\begin{aligned}
X_{0}^{-}= & \sum_{j=1}^{N}\left\{X_{0 j}(2 h k+i): h \geq 0,0 \leq i \leq k-1,-\frac{(4 h k+2 i+1) \pi}{2 k} \cot \frac{(2 i+1) \pi}{4 k}+\alpha_{j}<0\right\} \\
& \cup \sum_{j=1}^{N}\left\{X_{0 j}(2 h k+2 k-i-1):\right. \\
& \left.h \geq 0,0 \leq i \leq k-1, \frac{(4 h k+4 k-2 i-1) \pi}{2 k} \cot \frac{(2 i+1) \pi}{4 k}+\alpha_{j}<0\right\}, \\
X_{\infty}^{0}= & \sum_{j=1}^{N}\left\{X_{\infty j}(2 h k+i): h \geq 0,0 \leq i \leq k-1,-\frac{(4 h k+2 i+1) \pi}{2 k} \cot \frac{(2 i+1) \pi}{4 k}+\beta_{j}=0\right\} \\
& \cup \sum_{j=1}^{N}\left\{X_{\infty j}(2 h k+2 k-i-1):\right. \\
& \left.h \geq 0,0 \leq i \leq k-1, \frac{(4 h k+4 k-2 i-1) \pi}{2 k} \cot \frac{(2 i+1) \pi}{4 k}+\beta_{j}=0\right\}, \\
& N X_{0}^{N}\left\{X_{j=1}(2 h k+i): h \geq 0,0 \leq i \leq k-1,-\frac{(4 h k+2 i+1) \pi}{2 k} \cot \frac{(2 i+1) \pi}{4 k}+\alpha_{j}=0\right\} \\
& \cup \sum_{j=1}^{N}\left\{X_{0 j}(2 h k+2 k-i-1):\right. \\
& \left.0,0 \leq i \leq k-1, \frac{(4 h k+4 k-2 i-1) \pi}{2 k} \cot \frac{(2 i+1) \pi}{4 k}+\alpha_{j}=0\right\} . \\
& \\
&
\end{aligned}
$$

It is easy to see that $\operatorname{dim} X_{\infty}^{0}<\infty$ and $\operatorname{dim} X_{0}^{0}<\infty$.

Lemma 3.1 ([3], Lemma 2.4) Suppose $X$ is a Hilbert space, $\Phi: X \rightarrow R$ is a differentiable functional, and $L: X \rightarrow X$ is a linear operator. Then there are two closed $S^{1}$-invariant linear subspaces $X^{+}$and $X^{-}$such that

(a) $X^{+} \cup X^{-}$is closed and of finite codimension in $X$,

(b) $\widehat{L}\left(X^{-}\right) \subset X^{-}, \widehat{L}=L+P^{-1} A_{0}$ or $\widehat{L}=L+P^{-1} A_{\infty}$,

(c) there exists $c_{0} \in R$ such that

$$
\inf _{x \in X^{+}} \Phi(x) \geq c_{0}
$$

(d) there is $c_{\infty} \in R$ such that

$$
\Phi(x) \leq c_{\infty}<\Phi(0)=0, \quad \forall x \in X^{-} \cap S_{r}=\left\{x \in X^{-}:\|x\|=r\right\}
$$

(e) $\Phi$ satisfies the $(P . S)_{c}$-condition for $c_{0}<c<c_{\infty}$, that is, every $\left\{x_{n}\right\} \subseteq X$ satisfying $\Phi\left(x_{n}\right) \rightarrow c$ and $\Phi^{\prime}\left(x_{n}\right) \rightarrow 0$ has a convergent subsequence. Then $\Phi$ has at least $\frac{1}{2}\left[\operatorname{dim}\left(X^{+} \cap X^{-}\right)-\operatorname{codim}_{X}\left(X^{+} \cup X^{-}\right)\right]$generally different critical orbits in $\Phi^{-1}\left(\left[c_{0}, c_{\infty}\right]\right)$ if

$$
\left[\operatorname{dim}\left(X^{+} \cap X^{-}\right)-\operatorname{codim}_{X}\left(X^{+} \cup X^{-}\right)\right]>0 .
$$


Lemma 3.2 There exists $\sigma>0$ such that

$$
\left\langle\left(L+P^{-1} A_{\infty}\right) x, x\right\rangle>\sigma\|x\|^{2}, \quad x \in X_{\infty}^{+}
$$

and

$$
\left\langle\left(L+P^{-1} A_{\infty}\right) x, x\right\rangle<-\sigma\|x\|^{2}, \quad x \in X_{\infty}^{-} .
$$

Proof Let

$$
\begin{aligned}
X_{\infty j} & =\sum_{i=0}^{\infty} X_{\infty j}(i) \\
& =\left\{x(t)=\sum_{i=0}^{\infty}\left(a_{j} \cos \frac{(2 i+1) \pi t}{2 k}+b_{j} \sin \frac{(2 i+1) \pi t}{2 k}\right) v_{j}: a_{j}, b_{j} \in R, j=1,2, \ldots, N\right\} .
\end{aligned}
$$

Then $X=\sum_{j=1}^{N} X_{\infty j}$. We need to consider two cases, $\beta_{j} \geq 0$ and $\beta_{j}<0$. In the following part, we just give the proof for $\beta_{j} \geq 0$, as the other case is similar.

For $\beta_{j} \geq 0, i \in\{0,1, \ldots, k-1\}$, and $x \in X_{\infty j}$,

$$
-\frac{(4 h k+2 i+1) \pi}{2 k} \cot \frac{(2 i+1) \pi}{4 k}+\beta_{j}>-\frac{\left(4 h^{+}(i) k+2 i+1\right) \pi}{2 k} \cot \frac{(2 i+1) \pi}{4 k}+\beta_{j}>0,
$$

where $h^{+}(i)=\max \left\{h \in N:-\frac{(4 h k+2 i+1) \pi}{2 k} \cot \frac{(2 i+1) \pi}{4 k}+\beta_{j}>0\right\}$, and

$$
-\frac{(4 h k+2 i+1) \pi}{2 k} \cot \frac{(2 i+1) \pi}{4 k}+\beta_{j}<-\frac{\left(4 h^{-}(i) k+2 i+1\right) \pi}{2 k} \cot \frac{(2 i+1) \pi}{4 k}+\beta_{j}<0,
$$

where $h^{-}(i)=\min \left\{h \in N:-\frac{(4 h k+2 i+1) \pi}{2 k} \cot \frac{(2 i+1) \pi}{4 k}+\beta_{j}<0\right\}$.

Then we can choose

$$
\begin{aligned}
\sigma_{i}= & \min \left\{-\frac{\pi}{2 k} \cot \frac{(2 i+1) \pi}{4 k}+\frac{\beta_{j}}{4 h^{+}(i) k+2 i+1},\right. \\
& \left.\frac{\pi}{2 k} \cot \frac{(2 i+1) \pi}{4 k}-\frac{\beta_{j}}{4 h^{-}(i) k+2 i+1}\right\} \\
> & 0,
\end{aligned}
$$

and let $\sigma_{j}=\min \left\{\sigma_{0}, \sigma_{1}, \ldots, \sigma_{k-1}\right\}>0$, and then let $\sigma=\min \left\{\sigma_{j}: j=1,2, \ldots, N\right\}$. The proof is over.

Lemma 3.3 If $\left(f_{1}\right)$ and $\left(f_{2}\right)$ hold, then the functional $\Phi$ given by (2.1) satisfies the (P.S)condition.

Proof Let $\Pi, \Lambda$, and $\Gamma$ be the orthogonal mappings from $X$ to $X_{\infty}^{+}, X_{\infty}^{-}$, and $X_{\infty}^{0}$, respectively. From (1.3) we get

$$
\left|\left\langle P^{-1}\left(\nabla F(x)-A_{\infty} x\right), x\right\rangle\right|<\frac{\sigma}{2}\|x\|^{2}+\tilde{M}, \quad x \in X
$$

for some $\widetilde{M}>0$. 
Suppose that $\left\{x_{n}\right\} \subset X$ is a subsequence such that $\Phi^{\prime}\left(x_{n}\right) \rightarrow 0$ and $\Phi\left(x_{n}\right)$ is bounded. Let $w_{n}=\Pi x_{n}, y_{n}=\Lambda x_{n}$, and $z_{n}=\Gamma x_{n}$. Then

$$
\Pi\left(L+P^{-1} A_{\infty}\right)=\left(L+P^{-1} A_{\infty}\right) \Pi, \Lambda\left(L+P^{-1} A_{\infty}\right)=\left(L+P^{-1} A_{\infty}\right) \Lambda
$$

From

$$
\left\langle\Phi^{\prime}\left(x_{n}\right), x_{n}\right\rangle=\left\langle L x_{n}+P^{-1} \nabla F\left(x_{n}\right), x_{n}\right\rangle=\left\langle\left(L+P^{-1} A_{\infty}\right) x_{n}, x_{n}\right\rangle+\left\langle P^{-1}\left(\nabla F\left(x_{n}\right)-A_{\infty} x_{n}\right), x_{n}\right\rangle
$$

and (3.1) we have

$$
\begin{aligned}
\left\langle\Pi \Phi^{\prime}\left(x_{n}\right), x_{n}\right\rangle & =\left\langle\Pi\left(L+P^{-1} A_{\infty}\right) x_{n}, x_{n}\right\rangle+\left\langle\Pi P^{-1}\left(\nabla F\left(x_{n}\right)-A_{\infty} x_{n}\right), x_{n}\right\rangle \\
& =\left\langle\left(L+P^{-1} A_{\infty}\right) w_{n}, w_{n}\right\rangle+\left\langle\Pi P^{-1}\left(\nabla F\left(x_{n}\right)-A_{\infty} x_{n}\right), w_{n}\right\rangle \\
& >\frac{\sigma}{2}\left\|w_{n}\right\|^{2}-\tilde{M} .
\end{aligned}
$$

Then we get that $w_{n}$ is bounded. Similarly, $y_{n}$ is bounded. Meanwhile, from $\left(f_{2}\right)$ we get

$$
\begin{aligned}
\Phi\left(x_{n}\right)= & \frac{1}{2}\left\langle\left(L+P^{-1} A_{\infty}\right) x_{n}, x_{n}\right\rangle+\int_{0}^{4 k}\left(F\left(x_{n}\right)-\frac{1}{2}\left(A_{\infty} x_{n}, x_{n}\right)\right) d t \\
\geq & \frac{1}{2}\left\langle\left(L+P^{-1} A_{\infty}\right) w_{n}, w_{n}\right\rangle+\frac{1}{2}\left\langle\left(L+P^{-1} A_{\infty}\right) y_{n}, y_{n}\right\rangle \\
& +\int_{0}^{4 k} r\left(\left|x_{n}(t)\right|\right) d t-4 k M .
\end{aligned}
$$

Then $\left\|z_{n}\right\|$ is bounded since $\Phi\left(x_{n}\right)$ is bounded. So, $\left\|x_{n}\right\|$ is bounded.

Furthermore, from (2.4) we have

$$
\begin{aligned}
(\Pi+N) \Phi^{\prime}\left(x_{n}\right) & =(\Pi+\Lambda) L x_{n}+(\Pi+N) K\left(x_{n}\right) \\
& =L\left(w_{n}+y_{n}\right)+(\Pi+N) K\left(x_{n}\right) .
\end{aligned}
$$

Then we can suppose without loss of generality that $K\left(x_{n}\right) \rightarrow \eta$ because $K$ is compact and $x_{n}$ is bounded. Then

$$
\left.L\right|_{x_{\infty}^{+}+x_{\infty}^{-}}\left(w_{n}+y_{n}\right) \rightarrow-(\Pi+N) \eta
$$

Meanwhile, we can easily see that the dimension of $X_{\infty}^{0}$ is finite, so we can suppose that $z_{n} \rightarrow \varphi$ as $z_{n}$ is bounded. Hence

$$
x_{n}=z_{n}+w_{n}+y_{n} \rightarrow \varphi-\left(\left.L\right|_{x_{\infty}^{+}+x_{\infty}^{-}}\right)^{-1}(\Pi+\Lambda) \eta,
$$

and the (P.S)-condition is proved.

Lemma 3.4 If $x$ is a critical point of $\Phi$, then it is a solution to system (1.4). 
Proof Suppose $x$ is a critical point of $\Phi$ given by (2.1). Then $x(t)$ satisfies

$$
-\sum_{s=1}^{2 k-1} x^{\prime}(t-s)+\nabla F(x(t))=0, \quad \text { a.e. } t \in[0,4 k]
$$

Consequently,

$$
\begin{aligned}
& -\sum_{s=1}^{2 k-1} x^{\prime}(t-s-1)+\nabla F(x(t-1))=0, \\
& -\sum_{s=1}^{2 k-1} x^{\prime}(t-s-2)+\nabla F(x(t-2))=0, \\
& -\sum_{s=1}^{2 k-1} x^{\prime}(t-s-3)+\nabla F(x(t-3))=0, \\
& \vdots \\
& -\sum_{s=0}^{2 k-1} x^{\prime}(t-s-(2 k-1))+\nabla F(x(t-(2 k-1)))=0 .
\end{aligned}
$$

Calculating $\left(3.5 \_1\right)-\left(3.5 \_2\right)+\left(3.5 \_3\right)-\cdots+\left(3.5 \_(2 k-1)\right)$, we have

$$
x^{\prime}(t)+\sum_{s=1}^{2 k-1}(-1)^{s+1} \nabla F(x(t-s))=0, \quad \text { a.e. } t \in[0,4 k]
$$

that is,

$$
x^{\prime}(t)=-\sum_{s=1}^{2 k-1}(-1)^{s+1} \nabla F(x(t-s)), \quad \text { a.e. } t \in[0,4 k],
$$

and hence $x$ is a solution of (1.1).

\section{Main results}

Denote

$$
\begin{aligned}
& N\left(\alpha_{j}\right)= \begin{cases}-\sum_{i=0}^{k-1} \sharp\left\{h \geq 0: 0<\frac{(4 h k+4 k-2 i-1) \pi}{2 k} \cot \frac{(2 i+1) \pi}{4 k}<-\alpha_{j}\right\}, & \alpha_{j}<0, \\
\sum_{i=0}^{k-1} \sharp\left\{h \geq 0: 0<\frac{(4 h k+2 i+1) \pi}{2 k} \cot \frac{(2 i+1) \pi}{4 k}<\alpha_{j}\right\}, & \alpha_{j} \geq 0,\end{cases} \\
& N\left(\beta_{j}\right)= \begin{cases}-\sum_{i=0}^{k-1} \sharp\left\{h \geq 0: 0<\frac{(4 h k+4 k-2 i-1) \pi}{2 k} \cot \frac{(2 i+1) \pi}{4 k}<-\beta_{j}\right\}, & \beta_{j}<0, \\
\sum_{i=0}^{k-1} \sharp\left\{h \geq 0: 0<\frac{(4 h k+2 i+1) \pi}{2 k} \cot \frac{(2 i+1) \pi}{4 k}<\beta_{j}\right\}, & \beta_{j} \geq 0,\end{cases} \\
& N^{0}\left(\alpha_{j-}\right)=\sum_{i=0}^{k-1} \sharp\left\{h \geq 0: 0<\frac{(4 h k+4 k-2 i-1) \pi}{2 k} \cot \frac{(2 i+1) \pi}{4 k}=-\alpha_{j}\right\}, \quad \alpha_{j}<0, \\
& N^{0}\left(\alpha_{j+}\right)=\sum_{i=0}^{k-1} \sharp\left\{h \geq 0: 0<\frac{(4 h k+2 i+1) \pi}{2 k} \cot \frac{(2 i+1) \pi}{4 k}=\alpha_{j}\right\}, \quad \alpha_{j} \geq 0,
\end{aligned}
$$




$$
\begin{aligned}
& N^{0}\left(\beta_{j-}\right)=\sum_{i=0}^{k-1} \sharp\left\{h \geq 0: 0<\frac{(4 h k+4 k-2 i-1) \pi}{2 k} \cot \frac{(2 i+1) \pi}{4 k}=-\beta_{j}\right\}, \quad \beta_{j}<0, \\
& N^{0}\left(\beta_{j+}\right)=\sum_{i=0}^{k-1} \sharp\left\{h \geq 0: 0<\frac{(4 h k+2 i+1) \pi}{2 k} \cot \frac{(2 i+1) \pi}{4 k}=\beta_{j}\right\}, \quad \beta_{j} \geq 0,
\end{aligned}
$$

and

$$
\begin{array}{ll}
N\left(A_{\infty}\right)=\sum_{j=1}^{N} N\left(\beta_{j}\right), & N\left(A_{0}\right)=\sum_{j=1}^{N} N\left(\alpha_{j}\right), \\
N^{0}\left(A_{\infty-}\right)=\sum_{j=1}^{N} N^{0}\left(\beta_{j-}\right), & N^{0}\left(A_{\infty+}\right)=\sum_{j=1}^{N} N^{0}\left(\beta_{j+}\right), \\
N^{0}\left(A_{0-}\right)=\sum_{j=1}^{N} N^{0}\left(\alpha_{j-}\right), & N^{0}\left(A_{0+}\right)=\sum_{j=1}^{N} N^{0}\left(\alpha_{j_{+}}\right) .
\end{array}
$$

Theorem 4.1 System (1.1) has at least

$$
\begin{aligned}
n= & \max \left\{N\left(A_{\infty}\right)-N\left(A_{0}\right)-N^{0}\left(A_{\infty-}\right)-N^{0}\left(A_{0+}\right),\right. \\
& \left.N\left(A_{0}\right)-N\left(A_{\infty}\right)-N^{0}\left(A_{0_{-}}\right)-N^{0}\left(A_{\infty_{+}}\right)\right\} \\
> & 0
\end{aligned}
$$

4k-periodic orbits when $\left(f_{1}\right)$ and $\left(f_{2}\right)$ hold.

Proof Without loss of generality, we suppose

$$
n=N\left(A_{\infty}\right)-N\left(A_{0}\right)-N^{0}\left(A_{\infty-}\right)-N^{0}\left(A_{0+}\right) .
$$

Then letting $X^{+}=X_{\infty}^{+}$and $X^{-}=X_{0}^{-}$, we get

$$
X \backslash\left(X^{+} \cup X^{-}\right)=X \backslash\left(X_{\infty}^{+} \cup X_{0}^{-}\right) \subseteq X_{\infty}^{0} \cup X_{0}^{0} \cup\left(X_{\infty}^{+} \cap X_{0}^{-}\right) .
$$

Obviously,

$$
\operatorname{codim}_{X}\left(X^{+}+X^{-}\right) \leq \operatorname{dim} X_{\infty}^{0}+\operatorname{dim} X_{0}^{0}+\operatorname{dim}\left(X_{\infty}^{+} \cap X_{0}^{-}\right)<\infty
$$

which means that the codimension of $\left(X^{+} \cup X^{-}\right)$is finite. For each $x \in X(i)$, we have $(L+$ $\left.P^{-1} A_{\infty}\right) x \in X(i)$. The (PS)-condition is satisfied by Lemma 3.3. Moreover, from (1.3) we get $\left|F(x)-\frac{1}{2}\left(A_{\infty} x, x\right)\right|<\frac{1}{4} \sigma\|x\|^{2}+M_{1}, x \in R^{N}$, for some $M_{1}>0$, and from Lemma 3.2 we know that there exists $\sigma>0$ such that $\left\langle\left(L+P^{-1} A_{\infty}\right) x, x\right\rangle>\sigma\|x\|^{2}, x \in X_{\infty}^{+}$. Then

$$
\begin{aligned}
\Phi(x) & =\frac{1}{2}\left\langle\left(L+P^{-1} A_{\infty}\right) x, x\right\rangle+\int_{0}^{4 k}\left[F(x(t))-\frac{1}{2}\left(A_{\infty} x, x\right)\right] d t \\
& \geq \frac{1}{2} \sigma\|x\|^{2}-\frac{1}{4} \sigma\|x\|^{2}-4 k M_{1} \\
& \geq \frac{1}{4} \sigma\|x\|^{2}-4 k M_{1}
\end{aligned}
$$


for $x \in X^{+}$. Therefore there exists $c_{0} \in R$ such that

$$
\inf _{x \in X^{+}} \Phi(x) \geq c_{0}
$$

Similarly, we get that there exist $r, \sigma>0$ such that $\left|F(x)-\frac{1}{2}\left(A_{0} x, x\right)\right|<\frac{1}{4} \sigma\|x\|^{2},\|x\|=r$. Then

$$
\begin{aligned}
\Phi(x) & =\frac{1}{2}\left\langle\left(L+P^{-1} A_{0}\right) x, x\right\rangle+\int_{0}^{4 k}\left[F(x(t))-\frac{1}{2}\left(A_{0} x, x\right)\right] d t \\
& \leq-\frac{1}{2} \sigma\|x\|^{2}+\frac{1}{4} \sigma\|x\|^{2} \\
& \leq-\frac{1}{4} \sigma\|x\|^{2}
\end{aligned}
$$

for $x \in X^{-}$. This means that there exist $r>0$ and $c_{\infty}<0$, such that

$$
\Phi(x) \leq c_{\infty}<0=\Phi(0), \quad \forall x \in X^{-} \cap S_{r}=\{x \in X:\|x\|=r\} .
$$

On the other hand, for $i \in\{0,1, \ldots, k-1\}$,

$$
\begin{aligned}
\left\langle\left(L+P^{-1} A_{\infty}\right) x, x\right\rangle= & \left(-\frac{\pi}{2 k} \cot \frac{(2 i+1) \pi}{4 k}+\frac{\beta_{j}}{4 h k+2 i+1}\right)\|x\|^{2}, \quad x \in X(2 h k+i), \\
\left\langle\left(L+P^{-1} A_{\infty}\right) x, x\right\rangle= & \left(\frac{\pi}{2 k} \cot \frac{(2 i+1) \pi}{4 k}+\frac{\beta_{j}}{4 h k+4 k-2 i-1}\right)\|x\|^{2}, \\
& x \in X(2 h k+2 k-i-1),
\end{aligned}
$$

and

$$
\begin{aligned}
\left\langle\left(L+P^{-1} A_{0}\right) x, x\right\rangle= & \left(-\frac{\pi}{2 k} \cot \frac{(2 i+1) \pi}{4 k}+\frac{\alpha_{j}}{4 h k+2 i+1}\right)\|x\|^{2}, \quad x \in X(2 h k+i), \\
\left\langle\left(L+P^{-1} A_{0}\right) x, x\right\rangle= & \left(\frac{\pi}{2 k} \cot \frac{(2 i+1) \pi}{4 k}+\frac{\alpha_{j}}{4 h k+4 k-2 i-1}\right)\|x\|^{2}, \\
& x \in X(2 h k+2 k-i-1) .
\end{aligned}
$$

Hence we have that

$$
\begin{aligned}
& X_{\infty}^{+}(2 h k+i)=X_{\infty}^{+} \cap X(2 h k+i)=\emptyset, \\
& X_{0}^{-}(2 h k+2 k-i-1)=X_{0}^{-} \cap X(2 h k+2 k-i-1)=\emptyset, \\
& X_{0}^{-}(2 h k+i)=X_{0}^{-} \cap X(2 h k+i)=X(2 h k+i), \\
& X_{\infty}^{+}(2 h k+2 k-i-1)=X_{\infty}^{+} \cap X(2 h k+2 k-i-1)=X(2 h k+2 k-i-1),
\end{aligned}
$$

when $h \geq 0$ is large enough. So there is $M>0$ such that

$$
\operatorname{dim}\left(X_{\infty}^{+}(s) \cap X_{0}^{-}(r)\right)-\operatorname{codim}_{X}\left(X_{\infty}^{+}(s)+X_{0}^{-}(s)\right)=0, \quad s>M .
$$


Then

$$
\begin{aligned}
n & =\frac{1}{2}\left[\operatorname{dim}\left(X^{+} \cap X^{-}\right)-\operatorname{codim}_{X}\left(X^{+}+X^{-}\right)\right] \\
& =\frac{1}{2}\left[\operatorname{dim}\left(X_{\infty}^{+} \cap X_{0}^{-}\right)-\operatorname{codim}_{X}\left(X_{\infty}^{+}+X_{0}^{-}\right)\right] \\
& =\frac{1}{2} \sum_{s=0}^{M}\left[\operatorname{dim}\left(X_{\infty}^{+}(s) \cap X_{0}^{-}(s)\right)-\operatorname{codim}_{X(s)}\left(X_{\infty}^{+}(s)+X_{0}^{-}(s)\right)\right] \\
& =\frac{1}{2} \sum_{s=0}^{M}\left[\operatorname{dim} X_{\infty}^{+}(s)+\operatorname{dim} X_{0}^{-}(s)-2 N\right] \\
& =\frac{1}{2} \sum_{s=0}^{M}\left[\operatorname{dim} X_{\infty}^{+}(s)+\operatorname{dim} X_{0}^{-}(s)\right]-N(M+1) .
\end{aligned}
$$

Then we have

$$
\begin{aligned}
& \sum_{s=0}^{M} \operatorname{dim}\left(X_{\infty}^{+}(s)\right)=2\left\{\begin{array}{cc}
N\left(A_{\infty}\right) \\
+N \times \sharp\{2 h k+2 k-i-1: 0 \leq 2 h k+2 k-i-1 \leq M\}, & \beta_{j} \geq 0, \\
N\left(A_{\infty}\right)-N^{0}\left(A_{\infty-}\right) & \\
+N \times \sharp\{2 h k+2 k-i-1: 0 \leq 2 h k+2 k-i-1 \leq M\}, & \beta_{j}<0,
\end{array}\right. \\
& \sum_{s=0}^{M} \operatorname{dim}\left(X_{0}^{-}(s)\right)=2 \begin{cases}-N\left(A_{0}\right)-N^{0}\left(A_{0+}\right)+N \times \sharp\{2 h k+i: 0 \leq 2 h k+i \leq M\}, & \alpha_{j} \geq 0, \\
-N\left(A_{0}\right)+N \times \sharp\{2 h k+i: 0 \leq 2 h k+i \leq M\}, & \alpha_{j}<0,\end{cases}
\end{aligned}
$$

and

$$
\begin{aligned}
\sum_{s=0}^{M}\left[\operatorname{dim} X_{\infty}^{+}(s)+\operatorname{dim} X_{0}^{-}(s)\right]= & 2\left[N\left(A_{\infty}\right)-N\left(A_{0}\right)-N^{0}\left(A_{\infty-}\right)-N^{0}\left(A_{0+}\right)\right] \\
& +2 N(M+1) .
\end{aligned}
$$

Therefore

$$
n=N\left(A_{\infty}\right)-N\left(A_{0}\right)-N^{0}\left(A_{\infty-}\right)-N^{0}\left(A_{0+}\right) .
$$

Theorem 4.2 System (1.1) possesses at least

$$
n=N\left(A_{\infty}\right)-N\left(A_{0}\right)+N^{0}\left(A_{\infty+}\right)+N^{0}\left(A_{0-}\right)>0
$$

4k-periodic orbits when $\left(f_{1}\right),\left(f_{2}\right),\left(f_{3}^{+}\right)$, and $\left(f_{4}^{-}\right)$hold.

Proof Let $X^{+}=X_{\infty}^{+}+X_{\infty}^{0}$ and $X^{-}=X_{-}^{0}+X_{0}^{0}$. The verification of conditions (a), (b), (c), (d), and (e) is similar to Theorem 4.1, so we can assume that (4.1) still holds. Let $X_{\infty}^{0}(i)=$ 


$$
\begin{aligned}
& X_{\infty}^{0} \cap X(i) \text { and } X_{0}^{0}(i)=X_{0}^{0} \cap X(i) \text {. Then } \\
& n=\frac{1}{2} \sum_{i=0}^{M}\left[\operatorname{dim}\left(X_{\infty}^{+}(i) \cap X_{0}^{-}(i)\right)-\operatorname{codim}_{X(i)}\left(X_{\infty}^{+}(i)+X_{0}^{-}(i)\right)\right]+\left(\operatorname{dim} X_{\infty}^{0}+\operatorname{dim} X_{0}^{0}\right) \\
&=\frac{1}{2} \sum_{i=0}^{M}\left[\operatorname{dim} X_{\infty}^{+}(i)+\operatorname{dim} X_{0}^{-}(i)-2 N\right]+\left(\operatorname{dim} X_{\infty}^{0}+\operatorname{dim} X_{0}^{0}\right) \\
&=\frac{1}{2} \sum_{i=0}^{M}\left[\operatorname{dim} X_{\infty}^{+}(i)+\operatorname{dim} X_{0}^{-}(i)\right]-N(M+1)+\left(\operatorname{dim} X_{\infty}^{0}+\operatorname{dim} X_{0}^{0}\right) \\
&=N\left(A_{\infty}\right)-N\left(A_{0}\right)-N^{0}\left(A_{\infty-}\right)-N^{0}\left(A_{0_{+}}\right) \\
&+\left(N^{0}\left(A_{\infty+}\right)+N^{0}\left(A_{0-}\right)+N^{0}\left(A_{0_{+}}\right)+N^{0}\left(A_{0-}\right)\right) \\
&= N\left(A_{\infty}\right)-N\left(A_{0}\right)+N^{0}\left(A_{\infty+}\right)+N^{0}\left(A_{0_{-}}\right) .
\end{aligned}
$$

Theorem 4.3 System (1.1) possesses at least

$$
n=N\left(A_{0}\right)-N\left(A_{\infty}\right)+N^{0}\left(A_{0_{+}}\right)+N^{0}\left(A_{\infty-}\right)>0
$$

4k-periodic orbits when $\left(f_{1}\right),\left(f_{2}\right),\left(f_{3}^{-}\right)$, and $\left(f_{4}^{+}\right)$hold.

The proof is almost the same as that of Theorem 4.2, and we omit it.

\section{Example}

Assume that $F \in C^{1}\left(R^{2}, R\right)$ satisfies

$$
F(x)= \begin{cases}\frac{3 \pi}{2} x_{1}^{2}+\frac{\pi}{2} x_{2}^{2}+\left(2 x_{1}^{2}+x_{2}^{2}\right)^{\frac{2}{3}}, & |x| \gg 1, \\ \frac{\pi}{2} x_{1}^{2}-\frac{3 \pi}{2} x_{2}^{2}-3 x_{1}^{\frac{12}{5}}-x_{2}^{\frac{8}{3}}, & |x| \ll 1 .\end{cases}
$$

We are to discuss the multiplicity of 12-periodic solutions of the equation

$$
x^{\prime}(t)=-\sum_{s=1}^{5}(-1)^{s+1} \nabla F(x(t-s)) .
$$

In this case, $k=3, \alpha_{1}=\pi, \alpha_{2}=-3 \pi, \beta_{1}=3 \pi, \beta_{2}=\pi$. Then

$$
\begin{aligned}
N\left(\alpha_{1}\right)= & \sharp\left\{h \geq 0: 0<\frac{(12 h+1) \pi}{6} \cot \frac{\pi}{12}<\pi\right\} \\
& +\sharp\left\{h \geq 0: 0<\frac{(12 h+3) \pi}{6} \cot \frac{3 \pi}{12}<\pi\right\} \\
& +\sharp\left\{h \geq 0: 0<\frac{(12 h+5) \pi}{6} \cot \frac{5 \pi}{12}<\pi\right\}
\end{aligned}
$$

$=4$, 


$$
\begin{aligned}
N\left(\alpha_{2}\right)= & -\sharp\left\{h \geq 0: 0<\frac{(12 h+11) \pi}{6} \cot \frac{\pi}{12}<3 \pi\right\} \\
& -\sharp\left\{h \geq 0: 0<\frac{(12 h+9) \pi}{6} \cot \frac{3 \pi}{12}<3 \pi\right\} \\
& -\sharp\left\{h \geq 0: 0<\frac{(12 h+7) \pi}{6} \cot \frac{5 \pi}{12}<3 \pi\right\} \\
= & -7, \\
N\left(\beta_{1}\right)= & \sharp\left\{h \geq 0: 0<\frac{(12 h+1) \pi}{6} \cot \frac{\pi}{12}<3 \pi\right\} \\
& +\sharp\left\{h \geq 0: 0<\frac{(12 h+3) \pi}{6} \cot \frac{3 \pi}{12}<3 \pi\right\} \\
& +\sharp\left\{h \geq 0: 0<\frac{(12 h+5) \pi}{6} \cot \frac{5 \pi}{12}<3 \pi\right\} \\
= & 9,
\end{aligned}
$$

$$
\begin{aligned}
N\left(\beta_{2}\right)= & \sharp\left\{h \geq 0: 0<\frac{(12 h+1) \pi}{6} \cot \frac{\pi}{12}<\pi\right\} \\
& +\sharp\left\{h \geq 0: 0<\frac{(12 h+3) \pi}{6} \cot \frac{3 \pi}{12}<\pi\right\} \\
& +\sharp\left\{h \geq 0: 0<\frac{(12 h+5) \pi}{6} \cot \frac{5 \pi}{12}<\pi\right\}
\end{aligned}
$$

$=4$,

$$
\begin{aligned}
& N\left(A_{0}\right)=N\left(\alpha_{1}\right)+N\left(\alpha_{2}\right)=-3, \quad N\left(A_{\infty}\right)=N\left(\beta_{1}\right)+N\left(\beta_{2}\right)=13, \\
& N^{0}\left(\alpha_{+}\right)=N^{0}\left(\beta_{-}\right)=N^{0}\left(\alpha_{-}\right)=N^{0}\left(\beta_{+}\right)=0 .
\end{aligned}
$$

According to Theorem 4.2, we get that Eq. (5.1) has at least 16 different 12-periodic orbits satisfying $x(t-6)=-x(t)$.

\section{Acknowledgements}

The authors thank the referees for carefully reading the manuscript and for their valuable suggestions, which have significantly improved the paper.

\section{Funding}

The present research is supported by the National Science Foundations of China (No. 11601493).

\section{Availability of data and materials}

Data sharing not applicable to this paper as no datasets were generated or analyzed during the current study.

\section{Competing interests}

The authors declare that they have no competing interests.

\section{Authors' contributions}

All authors contributed equally and significantly in writing this paper. All authors read and approved the final manuscript.

\section{Author details}

${ }^{1}$ School of Electromechanical Engineering, Weifang Engineering Vocational College, Shandong, P.R. China. ${ }^{2}$ School of Mathematics and Statistics, Beijing Institute of Technology, Beijing, P.R. China.

\section{Publisher's Note}

Springer Nature remains neutral with regard to jurisdictional claims in published maps and institutional affiliations. 


\section{References}

1. Benci, V.: On critical point theory for indefinite functionals in the presence of symmetries. Trans. Am. Math. Soc 274(2), 533-572 (1982). https://doi.org/10.1090/S0002-9947-1982-0675067-X

2. Fannio, L:: Multiple periodic solutions of Hamiltonian systems with strong resonance at infinity. Discrete Contin. Dyn. Syst., Ser. A 3, 251-264 (1997). https://doi.org/10.3934/dcds.1997.3.251

3. Fei, G.: Multiple periodic solutions of differential delay equations via Hamiltonian systems (I). Nonlinear Anal. TMA 65, 25-39 (2006). https://doi.org/10.1016/j.na.2005.06.011

4. Fei, G.: Multiple periodic solutions of differential delay equations via Hamiltonian systems (II). Nonlinear Anal. TMA 65 , 40-58 (2006). https://doi.org/10.1016/j.na.2005.06.012

5. Ge, W.: Periodic solutions of the differential delay equation $x^{\prime}(t)=-f(x(t-1))$. Acta Math. Sin. Engl. Ser. 12, 113-121 (1996). https://doi.org/10.1007/BF02108151

6. Ge, W., Mawhin, J.: Positive solutions to boundary value problems for second order ordinary differential equations with singular nonlinearities. Results Math. 34(1-2), 108-119 (1998). https://doi.org/10.1007/BF03322042

7. Ge, W.: Existence of exactly $n+1$ simple 4-periodic solutions of the differential delay equation $\dot{x}(t)=-f(x(t-1))$. Acta Math. Sin. 10(1), 80-87 (1994). https://doi.org/10.1007/BF02561551

8. Ge, W.: Oscillatory periodic solutions of differential delay equations with multiple lags. Chin. Sci. Bull. 42(6), 444-447 (1997). https://doi.org/10.1007/BF02882587

9. Ge, W., Zhang, L.: Multiple periodic solutions of delay differential systems with $2 k-1$ lags via variational approach. Nonlinear Anal. TMA 36(9), 4925-4943 (2016). https://doi.org/10.3034/dcds.2016013

10. Li, J., He, X.: Multiple periodic solutions of differential delay equations created by asymptotically linear Hamiltonian systems. Nonlinear Anal. TMA 31, 45-54 (1998). https://doi.org/10.1016/S0362-546X(96)00058-2

11. Li, J., He, X.: Proof and generalization of Kaplan-Yorke's conjecture under the condition $f^{\prime}(0)>0$ on periodic solution of differential delay equations. Sci. China Ser. A, Math. 42(9), 957-964 (1999). https://doi.org/10.1007/BF02880387

12. Li, J., He, X., Liu, Z.: Hamiltonian symmetric group and multiple periodic solutions of differential delay equations. Nonlinear Anal. TMA 35, 457-474 (1999). https://doi.org/10.1016/S0362-546X(97)00623-8

13. Guo, Z., Yu, J.: Multiple results on periodic solutions to delay differential equations via critical point theory. J. Differ Equ. 218, 15-35 (2005). https://doi.org/10.1016/j.jde.2005.08.007

14. Guo, Z., Yu, J.: Multiple results on periodic solutions to higher dimensional differential equations with multiple delays. J. Dyn. Differ. Equ. 23, 1029-1052 (2011). https://doi.org/10.1007/s10884-011-9228-z

15. Kaplan, J., Yorke, J.: Ordinary differential equations which yield periodic solutions of differential delay equations. J. Math. Anal. Appl. 48, 317-324 (1974). https://doi.org/10.1016/0022-247X(74)90162-0

16. Li, L., Xue, C., Ge, W.: Periodic orbits to Kaplan-Yorke like differential delay equations with two lags of ratio $(2 k-1) / 2$ Adv. Differ. Equ. 2016, Article ID 247 (2016). https://doi.org/10.1186/s13662-016-0967-3

17. Nussbaum, R.: Periodic solutions of special differential delay equations: an example in nonlinear functional analysis Proc. R. Soc. Edinb., Sect. A, Math. 81(1-2), 131-151 (1978). https://doi.org/10.1017/S03082-10500010490

18. Mawhin, J., Willem, M.: Critical Point Theory and Hamiltonian Systems. Springer, New York (1989)

\section{Submit your manuscript to a SpringerOpen ${ }^{\circ}$ journal and benefit from:}

- Convenient online submission

- Rigorous peer review

- Open access: articles freely available online

- High visibility within the field

- Retaining the copyright to your article

Submit your next manuscript at $\boldsymbol{s p r i n g e r o p e n . c o m ~}$ 